\title{
Solar neutrino results from Super Kamiokande
}

\author{
Yuuki Nakano*, for the Super Kamiokande Collaboration \\ Kamioka Observatory, ICRR, The Univ. of Tokyo \\ Higashi-Mozumi 456, Kamioka-cho, Hida-city Gifu 506-1205, Japan. \\ E-mail: ynakano@km.icrr.u-tokyo.ac.jp
}

\begin{abstract}
Super-Kamiokande (SK), a 50 kton water Cherenkov detector in Japan, observes ${ }^{8} \mathrm{~B}$ solar neutrinos through neutrino-electron elastic scattering. SK searches for distortions of the solar neutrino energy spectrum caused by the edge of the MSW resonance in the core of the sun. It also searches for a day-night solar neutrino flux asymmetry induced by the matter in the Earth. The installation of new front-end electronics in 2009 marks the beginning of the 4th phase of SK (SK-IV). This phase achieved the lowest energy threshold thus far (3.5 MeV kinetic energy). SK observed solar neutrinos for $\sim 18$ years, that is about 1.5 solar activity cycles. Our analysis of a possible correlation between solar neutrino flux and 11 year activity cycle will be presented. The combined energy spectrum and the day-night solar neutrino flux asymmetry from SK-I to SK-IV will be presented. A global oscillation analysis using SK-I,II,III, and SK-IV data and combined with the results of other solar neutrino experiments as well as KamLAND reactor experiment has been carried out. The results of this global analysis will be presented as well.
\end{abstract}

The 34th International Cosmic Ray Conference,

30 July- 6 August, 2015

The Hague, The Netherlands

${ }^{*}$ Speaker. 


\section{Introduction}

Solar neutrino flux measurements from the Super Kamiokande (SK) [1] and the Sudbury Neutrino Observatory (SNO) [2] experiments provided direct evidence that the deficit of the solar neutrinos observed by the Homestake [3] and other solar neutrino experiments is the results of solar neutrino flavor conversion. While this solar neutrino flavor conversion is well described by neutrino oscillations, there is still no direct evidence for this to be so. It is possible that the flavor conversion is driven by some other mechanism.

However, based on the current model and parameters of solar neutrino oscillations, there are two testable signatures available for the SK experiment to look for. The first is the observation and precision measurement of the expected MSW resonance curve [4]. Based on the current best-fit oscillation parameters extracted using both solar neutrino and reactor anti-neutrino data, there is an expected characteristic energy dependence of the flavor conversion. Higher energy solar neutrino, such as ${ }^{8} \mathrm{~B}$ and hep neutrinos, undergo complete resonant conversion within the Sun, while lower energy solar neutrinos, such as $p p,{ }^{7} \mathrm{Be}$, pep, $\mathrm{CNO}$ and the lowest energy ${ }^{8} \mathrm{~B}$ neutrinos, only suffer from vacuum oscillations. After averaging the vacuum oscillations due to energy resolution, the survival probability for low energy electron flavor solar neutrinos must exceed 50\%, while the resonant conversion of the higher energy solar neutrinos within the Sun results in a survival probability of about $30 \%$. The transition between the vacuum dominated and solar resonance dominated oscillations should occur near $3 \mathrm{MeV}$, making ${ }^{8} \mathrm{~B}$ solar neutrinos the best choice when searching for the transition point within the energy spectrum.

The second solar neutrino oscillation signature comes from the effect of the terrestrial matter density. This effect can be tested directly by comparing the rate of solar neutrino interactions during the day-time to the the rate during the night-time, when the solar neutrinos have passed through the Earth. After being resonantly converted into the second mass eigenstate within the Sun, the neutrino electron flavor content will in general be enhanced when passing through the Earth. This will lead to an excess in the electron elastic scattering rate during the night-time, and hence a negative "day-night asymmetry"

$$
A_{D N}=\frac{r_{D}-r_{N}}{r_{\text {ave }}}
$$

where $r_{D}\left(r_{N}\right)$ is the average day-time (night-time) rate and $r_{a v e}\left(=\left(r_{D}+r_{N}\right) / 2\right)$ is the average rate. SK observes a wide range of ${ }^{8} \mathrm{~B}$ solar neutrinos, making it the prime detector to search for both of the solar neutrino oscillation signatures.

\section{Super Kamiokande}

SK is a 50 kton imaging water Cherenkov detector, and it is located $1000 \mathrm{~m}$ underground (2700 $\mathrm{m}$ of water equivalent) in the Kamioka mine in Gifu prefecture, Japan. The experiment started taking data in April 1996.

Cherenkov light generated by charged particles scattered by neutrinos in water is detected by 11129 20-inch PMTs. SK measures the energy and direction of recoil electrons of solar neutrinoelectron elastic scattering, $v+e \rightarrow v+e$, and records the time of the interaction. 
The most important progress for the solar neutrino measurement was a reduction of the background thanks to improvements in the SK water system.

\section{Data analysis}

After installation of the new front-end electronics [5], physics data taking resumed on October 6th, 2008. the results presented here include data taken through the end of January 31st, 2014 (1669 days).

With various improvements to calibration methods [6] and analysis technique, the solar energy threshold could be lowered to $3.5 \mathrm{MeV}$ recoil electron kinetic energy.

\subsection{Flux}

As a special case for the flux analysis, additional data is included till the end of March 31st 2015, which is totally 2034 days.

In order to obtain the number of solar neutrino interactions, an extended maximum likelihood fit is used. This method was also used in the previous SK analyses [1, (7) B]. By this method, a total of about $77 \mathrm{k}$ solar neutrinos were observed throughout all SK phases.

SK-IV took data with the low energy threshold of $3.5 \mathrm{MeV}$. A clear solar neutrino signal in the $3.5-4.0 \mathrm{MeV}$ energy region is seen at about 8.2 $\sigma$.

The systematic uncertainties for the total flux in SK-IV were calculated using the same method as for SK-III [8]. The total systematic uncertainty for the SK-IV flux was found to be $\pm 1.7 \%$, improved from ${ }_{-3.2}^{+3.5} \%$ in SK-I [1] and $\pm 2.2 \%$ in SK-III [8], and the best value among all phases.

The flux results through all SK phases are summarized in the Table1 1 and are also shown in the left panel of Figure 1. Their measured ${ }^{8} \mathrm{~B}$ solar neutrino fluxes are consistent within uncertainties.

Table 1: Summary of the key features of each of SK. The energy threshold means recoil electron kinetic energy in MeV. The first error of the flux result is statistical and the second is systematic.

\begin{tabular}{ccccc}
\hline \hline & Live time[days] & Energy Range[MeV] & Flux $\left[\times 10^{6} / \mathrm{cm}^{2} / \mathrm{sec}\right]$ & Ref. \\
\hline SK-I & 1496 & $4.5-19.5$ & $2.38 \pm 0.02 \pm 0.08$ & [1] \\
SK-II & 791 & $6.5-19.5$ & $2.41 \pm 0.05_{-0.15}^{+0.16}$ & [7] \\
SK-III & 548 & $4.0-19.5$ & $2.40 \pm 0.04 \pm 0.05$ & [8] \\
SK-IV & $2034(1669)$ & $3.5-19.5$ & $2.31 \pm 0.02 \pm 0.04$ & - \\
\hline
\end{tabular}

These four measurements can be combined together to give the total SK flux of

$$
\Phi_{8_{\mathrm{B}}}(\text { Combined })=\{2.341 \pm 0.044\} \times 10^{6} / \mathrm{cm}^{2} / \mathrm{sec} .
$$

The solar activity cycle is the 11 years periodic change of sun spots releasing the magnetic flux at the surface of the Sun. They are strongly correlated with the solar activity cycle. SK observed solar neutrinos for 18 years, that is through about 1.5 solar activity cycles.

The right panel of Figure 1 shows the SK yearly flux measured throughout the different phases of SK together with the corresponding sun spot number. Using the present data, the $\chi^{2}$ was calculated with total experimental error as $\chi^{2}=13.10 / 18$ d.o.f., which corresponds to a probability of 

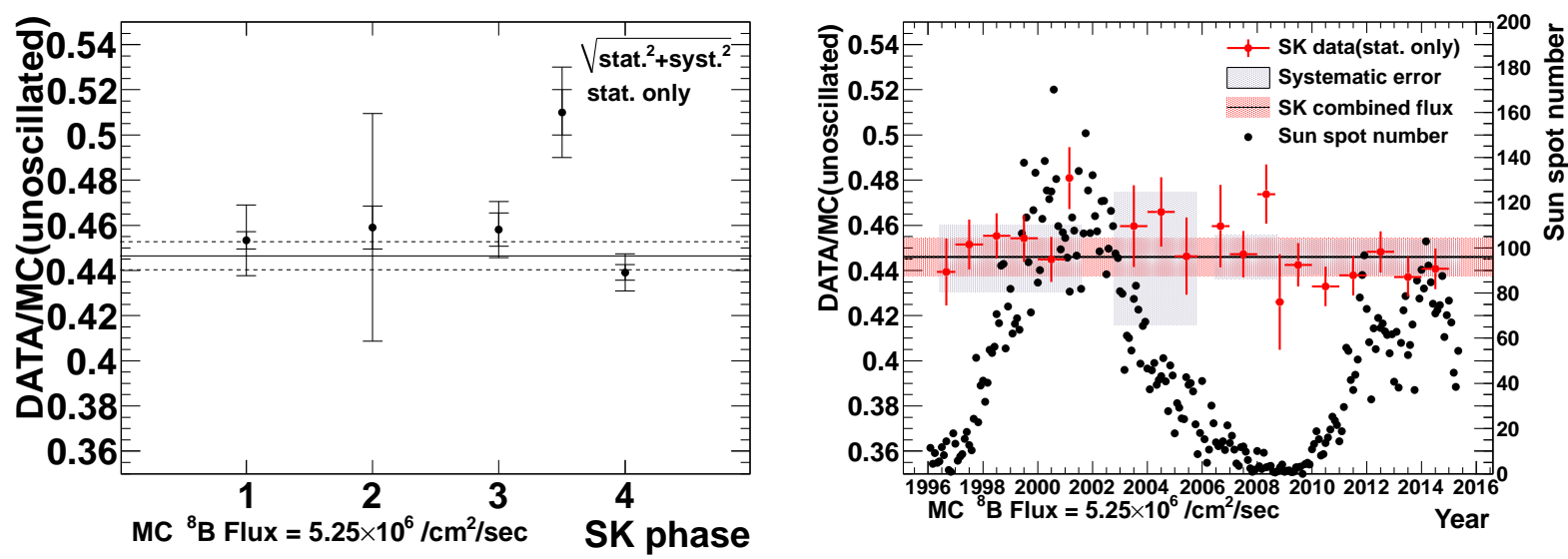

Figure 1: Left: Solar neutrino flux measurements from SK-I to SK-IV with statistical (inner) and systematic (outer) errors, normalized to the unoscillated MC prediction using $5.25 \times 10^{6} \mathrm{~cm}^{-2} \mathrm{sec}^{-1}$. The solid line shows the average among all SK phases. The large uncertainty during the SK-II phase was due to half the number of PMTs and correspondingly reduced sensitivity compared to other phases. Right: Solar neutrino yearly flux. The red points show the yearly flux measured by SK (statistical error only), the gray bands show the systematic error for each SK phase, the red band shows the error on the combined solar neutrino flux and the black points show the sun spot number. The sun spot number is cited from http://solarscience.msfc.nasa.gov/greenwch/spot_num.txt.

78.6\%. SK solar rate measurements are fully consistent with a constant solar neutrino flux emitted by the Sun.

\subsection{Energy spectrum}

The neutrino energy spectrum shape analysis consists of a combined fit to SK-I, II, and III data as well as 1669 days of SK-IV data to two generic functions as survival probability as a function of neutrino energy,

$$
\begin{aligned}
& P_{e e}\left(E_{V}\right)=c_{0}+c_{1}\left(\frac{E_{V}}{M e V}-10\right)+c_{2}\left(\frac{E_{V}}{M e V}-10\right)^{2} \text { (quadratic), } \\
& P_{e e}\left(E_{V}\right)=e_{0}+\frac{e_{1}}{e_{2}}\left(\exp \left(e_{2}\left(\frac{E_{v}}{M e V}-10\right)\right)-1\right) \text { (exponential). }
\end{aligned}
$$

The fit takes correlations between SK phases and energy bins into account.

The left panel of Figure 2 shows the energy spectrum measured by SK-IV which is expressed as the ratio of the observed elastic scattering rates over $\mathrm{MC}$ simulated expectation. Here, the ${ }^{8} \mathrm{~B}$ flux was constrained by SNO's NC measurement [9]. The right panel of Figure 2 shows the statistical combination of the four phases of SK, along with the best-fit coming from the generic quadratic (exponential) function fits in black (orange). Also shown in green (blue) is the expected MSW resonance curve assuming the best-fit neutrino oscillation parameters coming from a fit to SK data (all solar neutrino plus KamLAND data). SK spectrum results slightly disfavor the MSW resonance curves, but are consistent with the MSW resonance prediction within $1.0-1.7 \sigma$.

The left panel of Figure 3 shows the $1 \sigma$ allowed band of the survival probability $\left(P_{e e}\left(E_{v}\right)\right)$ as a function of neutrino energy. The right panel of Figure 3 shows the SK plus SNO combined 

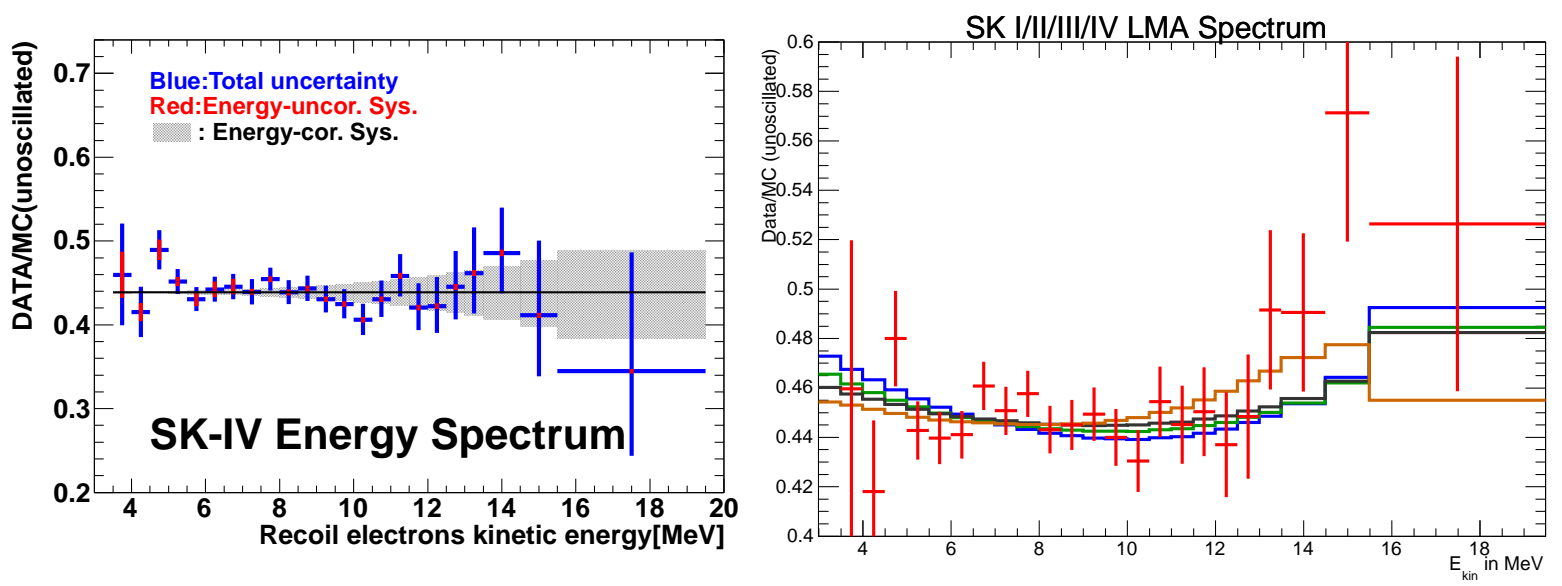

Figure 2: Left: The solar neutrino energy spectrum in SK-IV. Each point shows the ratio of the data to the expected flux using a non-oscillated ${ }^{8} \mathrm{~B}$ solar neutrino spectrum. Right: The energy spectrum combining SKI to SK-IV with prediction for (1) $\sin ^{2} \theta_{12}=0.308$ and $\Delta m_{21}^{2}=7.50 \times 10^{-5} \mathrm{eV}^{2}$ (blue), (2) $\sin ^{2} \theta_{12}=0.311$ and $\Delta m_{21}^{2}=4.85 \times 10^{-5} \mathrm{eV}^{2}$ (green), (3)quadratic fit (black) and (4)exponential fit (orange).

$1 \sigma$ quadratic fit band of the survival probability with the other experimental results ( $p p$ and CNO neutrino flux constraints from the all solar neutrino data [3 10], and the ${ }^{7} \mathrm{Be}$, pep and ${ }^{8} \mathrm{~B}$ flux measurements of Borexino [11]). The results are consistent with the transition curve expected for neutrino oscillation.
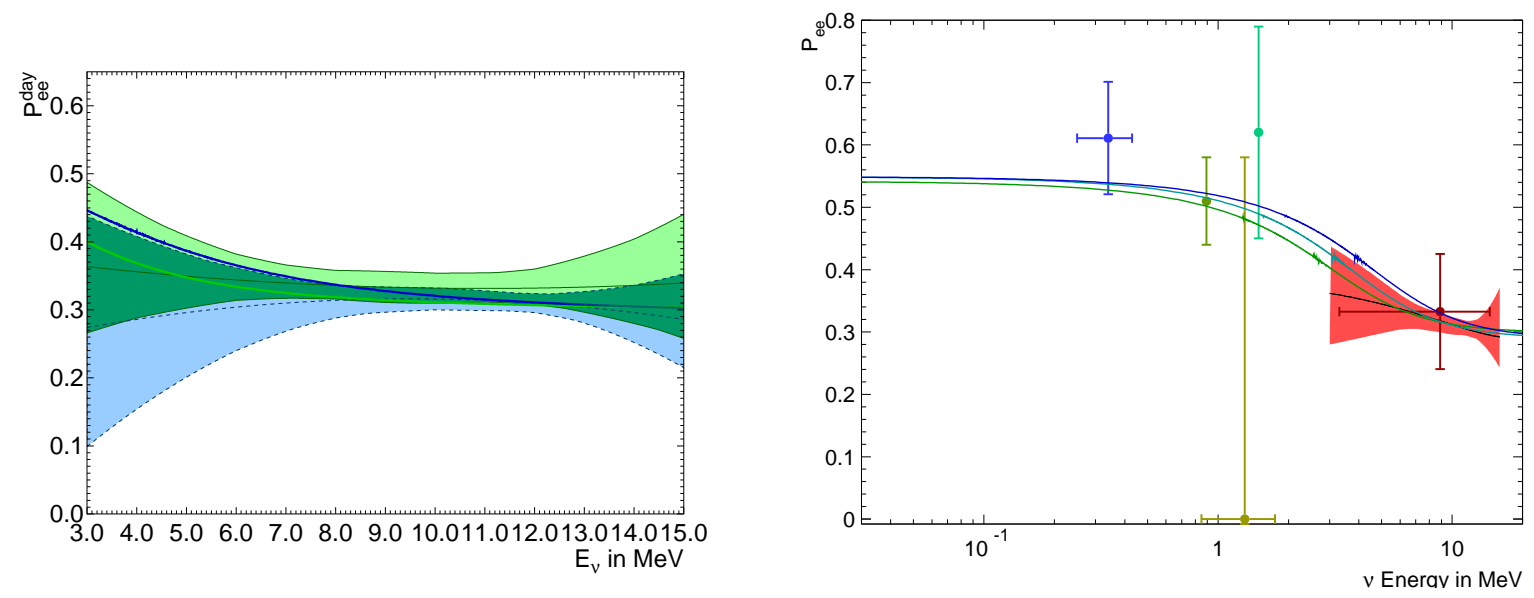

Figure 3: Left: Allowed survival probability $1 \sigma$ band from SK data (solid green) and SNO (dotted blue) data. Also shown are predictions based on the oscillation parameter of a fit to all solar data (green line) and a fit to all solar plus KamLAND data (blue line). Right: The $1 \sigma$ band from the combined data of SK and SNO is shown in red. Also shown are measurements of the ${ }^{7} \mathrm{Be}$ (green point), the pep (light green point) and the ${ }^{8} \mathrm{~B}$ flux (red point) by Borexino [11], as well as $p p$ (blue point) and CNO values (gold point) extracted from other experiments [10]. 


\subsection{Day/Night flux difference}

With the current best oscillation parameters, the day-night flux asymmetry 1.1) is expected to be about 3\% in the SK energy region. By comparing the separately measured day and night fluxes, the measured day-night asymmetries were calculated.

In our data analysis, a more sophisticated method used in searching for the day-night asymmetry employs a maximum likelihood fit to the amplitude of the expected variation to the entire data sample. The detail of this analysis is discussed in [12]. The overall amplitude using the combined SK period is summarized in the Table 2 The day-night fit amplitude is obtained by fitting the observed time variation during night with its expected variation, meaning that $A_{D N}^{\text {fit }}$ depends on the assumed value of $\Delta m_{21}^{2}$. The results gives $(-3.1 \pm 1.0 \pm 0.5) \%$ for fit with solar global plus KamLAND. This result deviates from zero by $2.8 \sigma$. The right panel of Figure 4 shows $A_{D N}^{\text {fit }}$ as a function of $\Delta m_{21}^{2}$ together with the expectation.

Table 2: Day-night asymmetry for all SK phases, coming from separate day and night rate measurements (2nd left column) and the amplitude fit (right two column). The uncertainties shown are statistical and systematic, respectively.

\begin{tabular}{cccc}
\hline \hline & $\begin{array}{c}\left.A_{D N} \pm \text { (stat. }\right) \pm \text { (syst.) } \\
\text { Equation 11.1) }\end{array}$ & $\begin{array}{c}\left.A_{D N}^{\text {fit }} \pm \text { (stat. }\right) \pm(\text { syst. }) \\
\Delta m_{21}^{2}=4.84 \times 10^{-5} \mathrm{eV}^{2}\end{array}$ & $\begin{array}{c}\left.A_{D N}^{\text {fit }} \pm \text { (stat. }\right) \pm(\text { syst. }) \\
\Delta m_{21}^{2}=7.50 \times 10^{-5} \mathrm{eV}^{2}\end{array}$ \\
\hline SK-I & $(-2.1 \pm 2.0 \pm 1.3) \%$ & $(-2.0 \pm 1.8 \pm 1.0) \%$ & $(-1.9 \pm 1.7 \pm 1.0) \%$ \\
SK-II & $(-5.5 \pm 4.2 \pm 3.7) \%$ & $(-4.4 \pm 3.8 \pm 1.0) \%$ & $(-4.4 \pm 3.6 \pm 1.0) \%$ \\
SK-III & $(-5.9 \pm 3.2 \pm 1.3) \%$ & $(-4.2 \pm 2.7 \pm 0.7) \%$ & $(-3.8 \pm 2.6 \pm 0.7) \%$ \\
SK-IV & $(-4.9 \pm 1.8 \pm 1.4) \%$ & $(-3.6 \pm 1.6 \pm 0.6) \%$ & $(-3.3 \pm 1.5 \pm 0.6) \%$ \\
\hline Combined & $(-4.1 \pm 1.2 \pm 0.8) \%$ & $(-3.3 \pm 1.0 \pm 0.5) \%$ & $(-3.1 \pm 1.0 \pm 0.5) \%$ \\
non-zero significance & $2.8 \sigma$ & $3.0 \sigma$ & $2.8 \sigma$ \\
\hline \hline
\end{tabular}

\subsection{Neutrino oscillation analysis}

A global solar neutrino oscillation analysis has been performed including all SK data as well as the results from SNO [9], the radiochemical experiments [10] and the ${ }^{7} \mathrm{Be}$ flux measurement from Borexino [11]. This analysis was compared to and combined with the reactor neutrino results from KamLAND [13].

The left panel of Figure 5 shows the allowed region of neutrino oscillation parameters in the $\Delta m_{21}^{2}$ and $\sin ^{2} \theta_{12}$ plane assuming $\sin ^{2} \theta_{13}$ is constrained by $\left\{\left(\sin ^{2} \theta_{13}-0.0242\right) / 0.0026\right\}^{2}$. The obtained parameters from the global solar analysis are $\Delta m_{21}^{2}=4.85_{-0.59}^{+1.40} \times 10^{-5} \mathrm{eV}^{2}$ and $\sin ^{2} \theta_{12}=$ $0.311 \pm 0.014$. Comparing these values with those from KamLAND, there is a $2 \sigma$ tension in the $\Delta m_{21}^{2}$ results. Combining the global solar data with KamLAND, the oscillation parameters become $\Delta m_{21}^{2}=7.50_{-0.18}^{+0.19} \times 10^{-5} \mathrm{eV}^{2}$ and $\sin ^{2} \theta_{12}=0.308 \pm 0.013$.

The right panel of Figure 5 shows the results of the $\theta_{13}$ unconstrained fit. The significance of non-zero $\theta_{13}$ from the solar plus KamLAND data combined fit is about $2.0 \sigma$, measured as $\sin ^{2} \theta_{13}=0.027_{-0.014}^{+0.016}$ and quite consistent with reactor anti-neutrino measurements [14]. 

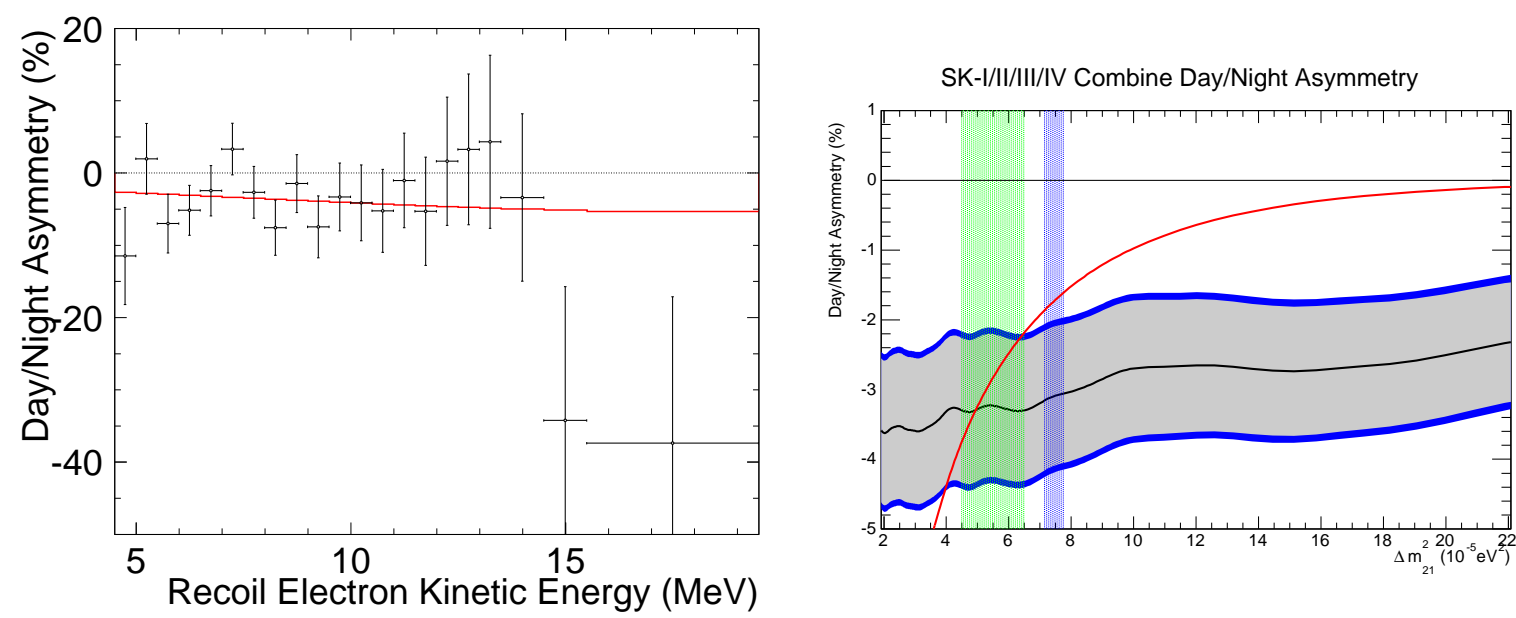

Figure 4: Left: SK day-night amplitude fit as a function of recoil electron kinetic energy, shown as the measured amplitude times the expected day-night asymmetry, for oscillation parameters chosen according to the SK best-fit. The error bars shown are statistical uncertainty only and the expected dependence for the best fit is shown in red. Right: Dependence of the measured day-night asymmetry (fitted day-night amplitude times the expected day-night asymmetry (red)) on $\Delta m_{21}^{2}$, for $\sin ^{2} \theta_{12}=0.311$ and $\sin ^{2} \theta_{13}=0.025$. The $1 \sigma$ stat (stat.+syst.) uncertainties are given by the light gray (blue) band. Overlaid are the $1 \sigma$ allowed ranges from the solar global fit (green vertical band) and the KamLAND experiment (blue vertical band).

\section{Summary}

Super Kamiokande has precisely measured the ${ }^{8} \mathrm{~B}$ solar neutrino flux, its time variation and recoil electron spectrum. When combined with the results from the previous three phases, the SK combined flux is $2.341 \pm 0.044$ (stat. + syst.) $\times 10^{6} / \mathrm{cm}^{2} / \mathrm{sec}$. SK data provided the first indication (at $2.8 \sim 3.0 \sigma$ ) of terrestrial matter effects on ${ }^{8} \mathrm{~B}$ solar neutrino oscillation. This is the first observation using a single detector and an identical neutrino beam with and without intervening matter to show that matter affects neutrino oscillation. SK gives the world's strongest constraints on the shape of the survival probability $P_{e e}\left(E_{v}\right)$ in the transition region between vacuum oscillations and the MSW resonance.

In the solar neutrino oscillation analysis combined with the KamLAND result, oscillation parameters are determined as $\Delta m_{21}^{2}=7.50_{-0.18}^{+0.19} \times 10^{-5} \mathrm{eV}^{2}, \sin ^{2} \theta_{12}=0.308 \pm 0.013$. When the constrain of $\theta_{13}$ is removed, solar neutrino experiments and KamLAND measure $\sin ^{2} \theta_{13}=$ $0.027_{-0.014}^{+0.016}$, a value is good agreement with reactor anti-neutrino measurements.

\section{References}

[1] J. Hosaka et al., Phys. Rev. D 73, 112001 (2006).

[2] Q. R. Ahmad, Phys. Rev. Lett. 87, 071301 (2001).

[3] R. Davis, Jr., D. S. Harmer, and K. C. Hoffman et al., Phys. Rev. Lett. 20, 1205 (1968).

[4] S. P. Mikheyev and A. Y. Smirnov, Sov. Jour. Nucl. Phys. 42, 913 (1985);

L. Wolfenstein, Phys. Rev. D 17, 2369 (1978). 

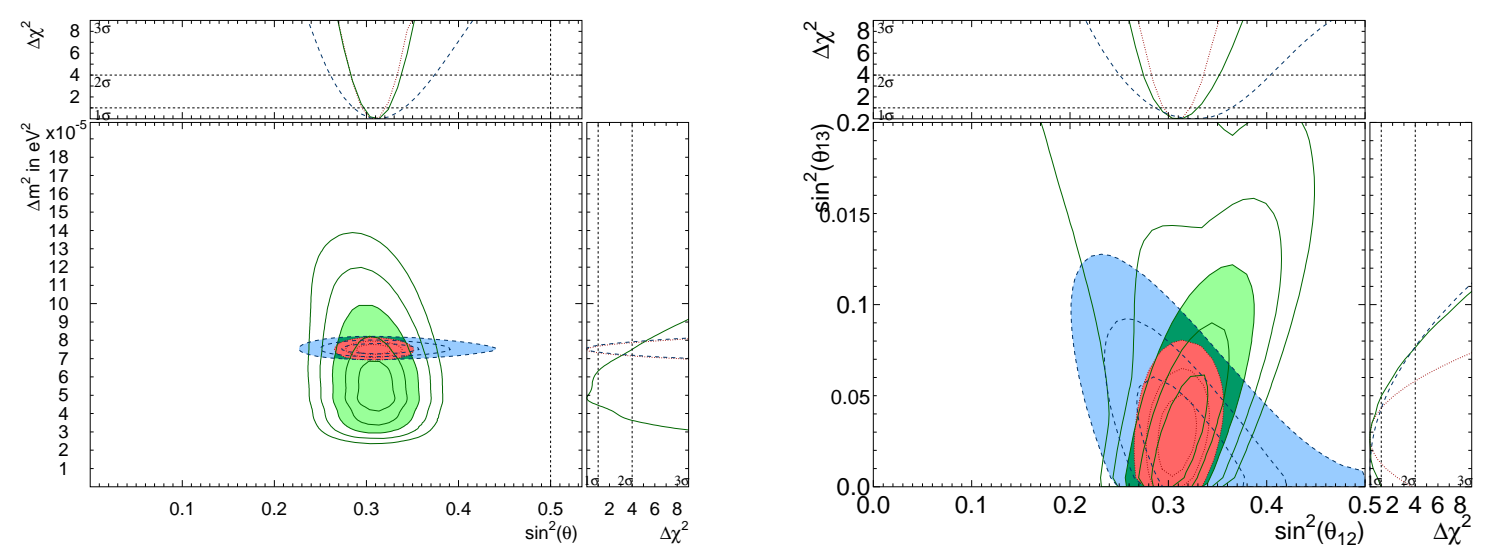

Figure 5: Left: Allowed contours for $\Delta m_{21}^{2}$ vs. $\sin ^{2} \theta_{12}$ from solar neutrino data (green) at 1, 2, 3, 4 and 5 $\sigma$ and KamLAND data (blue) at the 1,2 and $3 \sigma$ confidence level. Also shown is the combined result in red. For comparison, the almost identical result of the SK plus SNO combined fit is shown by the dashed dotted lines. The filled regions give the $3 \sigma$ confidence levels. Right: Allowed contours of $\sin ^{2} \theta_{13}$ vs. $\sin ^{2} \theta_{12}$, colors are the same in as the left panel.

[5] H. Nishino et al., Nucl. Inst. and Meth. A 620 (2009).

[6] K. Abe et al., Nucl. Inst. and Meth. A 737 C (2014).

[7] J. P. Cravens et al., Phys. Rev. D 78, 032002 (2008).

[8] K. Abe et al., Phys. Rev. D 83, 052010 (2011).

[9] B. Aharmin et al., Phys. Rev. C 88, 025501 (2013).

[10] J. N. Adburashitov et al., (SAGE collaboration), Phys. Rev. C 80, 015807 (2009);

M. Altmann et al., (GALLEX Collaboration), Phys. Lett. B 616, 174 (2005);

G. Bellini et al., (Borexino Collaboration), Phys. Rev. Lett. 107, 141302 (2011).

[11] Bellini et al., (Borexino Collaboration), Phys. Rev. D 82, 033006 (2010);

Bellini et al., (Borexino Collaboration), Phys. Rev. Lett. 707, 051302 (2012).

[12] M. B. Smy et al., Phys. Rev. D 69, 011104(R) (2004);

A. Renshaw et al., Phys. Rev. Lett. 112, 091805 (2014).

[13] S. Abe et al., Phys. Rev. Lett 100, 221803 (2008);

The KamLAND collaboration, arxiv:1303.4667v2 (2013).

[14] F. P. An et al. (Daya Bay Collaboration), Chin. Phys. C37, 011001 (2013);

J. K. Ahn et al. (RENO Collaboration), Phys. Rev. Lett. 108, 191802 (2012);

Y. Abe et al. (Double Chooz Collaboration), Phys. Rev. D86, 052008 (2012); 